This is a postprint version of the following published document:

Sánchez, R., Hernández, J. A. \& Larrabeiti, D. (2013). Using transparent WDM metro rings to provide an out-of-band control network for OpenFlow in MAN. 2013 15th International Conference on Transparent Optical Networks (ICTON), Cartagena (España), pp. 1-4.

DOI: $10.1109 /$ ICTON.2013.6602915

(C) 2013 IEEE. Personal use of this material is permitted. Permission from IEEE must be obtained for all other users, including reprinting/ republishing this material for advertising or promotional purposes, creating new collective works for resale or redistribution to servers or lists, or reuse of any copyrighted components of this work in other works 


\title{
Using Transparent WDM Metro Rings to Provide an Out-of-Band Control Network for OpenFlow in MAN
}

\author{
Rafael Sánchez, José Alberto Hernández, David Larrabeiti \\ Universidad Carlos III de Madrid, Spain \\ Avda. Universidad 30, E-28911 Leganés, Madrid, Spain \\ Tel. (+34) 91624 8459,Fax: (+34) 91624 8749,e-mail: \{rsfuente, jahgutie, dlarra\}@it.uc3m.es
}

\begin{abstract}
OpenFlow is a protocol that enables networks to evolve and change flexibly, by giving a remote controller the capability of modifying the behavior of network devices. In an OpenFlow network, each device needs to maintain a dedicated and separated connection with a remote controller. All these connections can

be described as the OpenFlow control network, that is the data network which transports control plane information, and can be deployed together with the data infrastructure plane (in-band) or separated (out-ofband), with advantages and disadvantages in both cases. The control network is a critical subsystem since the communication with the controller must be reliable and ideally should be protected against failures. This paper proposes a novel ring architecture to efficiently transport both the data plane and an out-of-band control network.
\end{abstract}

Keywords: OpenFlow, SDN, Metro Ethernet, Optical WDM rings.

\section{INTRODUCTION}

Software-defined networking (SDN) [1] is a new networking paradigm where the control and the data plane of a switch are decoupled. In SDN-based networks, the control functionality is performed by a centralised controller, which translates its decisions into forwarding rules sent to the individual devices. OpenFlow [2] is a standard protocol that provides an open communication interface between the control and the forwarding layers of network devices. Thanks to OpenFlow, the controller is capable of remotely manage all the switch forwarding rules. Each rule is translated into a forwarding entry, which comprises six fields: a matching rule, a set of actions, a number of counters, a priority value, a timeout value, and a cookie. OpenFlow operates as follows: when a packet arrives at a switch, the matching rule is evaluated. In the case of a positive match, the action is performed. Typical actions are: forwarding the packet to an output port, flooding the packet, dropping the packet, modifying a field, etc. If no match is found, then the packet is forwarded to the controller, which will handle it.

The SDN paradigm has a number of promising benefits, namely [3]: simple and inexpensive hardware, vendor neutrality, future-proof and flexibility. SDN has found its particular niche of application in the context of data center and campus networks [4]. In addition, some authors have recently proposed its suitability in carriergrade scenarios with the following extra benefits: energy efficiency [5] and reusability of the operational model of TDM.

This article provides a new SDN-based architecture for the metropolitan area network with WDM equipment reuse. In the past, metro solutions were implemented with SDH technologies. However, such an approach has been progressively replaced by all-optical transparent WDM-based networks, mainly thanks to their lower cost, simplicity and no electronic processing bottleneck [5][6][7][8]. The proposed architecture is based on the socalled tuneable-transmitter fixed-receiver (TT-FR) optical WDM rings, whereby each node in the ring is provided with a tuneable laser and a fixed receiver. Essentially, each node in the ring has a dedicated (fixed) wavelength for reception (dedicated home channel), and a tuneable laser that allows it to transmit data on the dedicated home channels of other nodes. Transparency is achieved because all nodes bypass all wavelengths except their dedicated home channel, which is captured and retrieved by its owner. To avoid collisions in the home wavelength of a node, a number of MAC protocols have been defined to arbitrate channel access, essentially based on multi-token passing (MTIT), wavelength inspection (DBORN), timeslot reservation (MAWSON, RingO), or a dedicated control channel (HORNET). Details about them can be found in reference [6] [Ch.13]. The main contribution of our proposal is to combine the advantages of TT-FR rings, which minimise the number of OEO conversions in the data plane, together with an out-of-band control network using an extra wavelength.

The paper is structured as follows: Section 2 describes four metro architecture options employing OpenFlow over TT-FR ring architectures; Section 3 overviews their advantages and disadvantages; finally Section 4 provides a summary of this article and some future work. 


\section{ARCHITECTURE OPTIONS}

Figure 1 shows four metro reference architectures, with a focus on the control network. We define such a control network as the IP data network required to communicate the switches with the centralised controller. This data network is used basically to transport TCP (SSH) connections between each switch and the controller.

The control network can be implemented as either in-band or out-of-band. In general terms, the main advantage of an in-band control network is its simplicity, since the same infrastructure can be used for both data and control networks. However, this approach lacks from separated protection against failures, since an eventual failure in the data plane may affect the control network, which needs to recover first (and this may imply a nonnegligible routing convergence time) before the controller can actually repair the data plane. The advantage of the out-of-band solution is that link failures in the data plane do not affect the control network (although this actually depends on the implementation), however it has the extra cost of additional hardware.

(a)

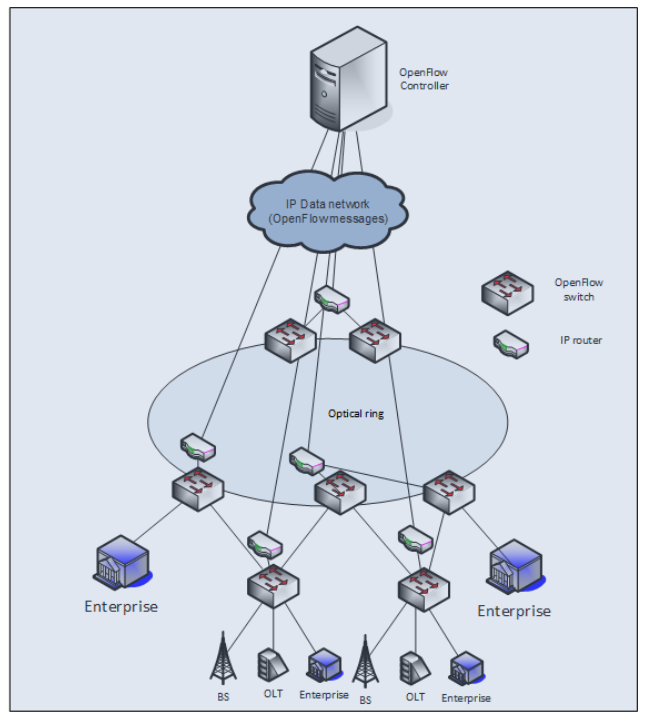

(c)

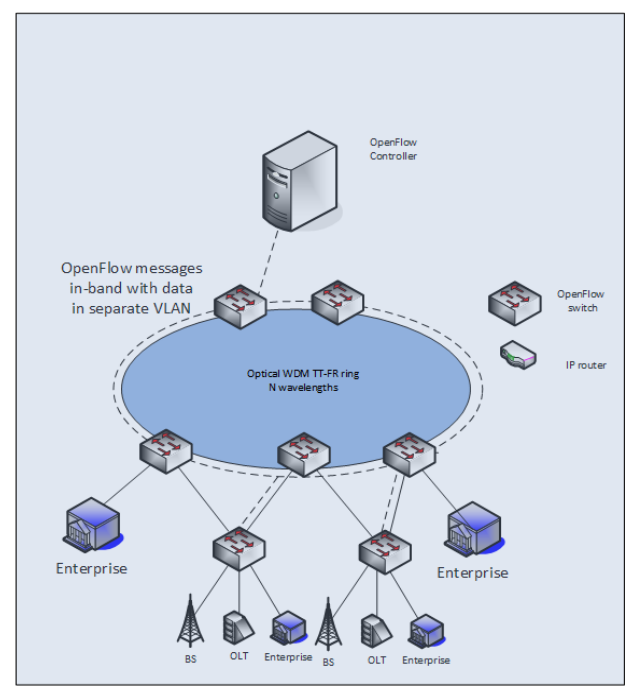

(b)

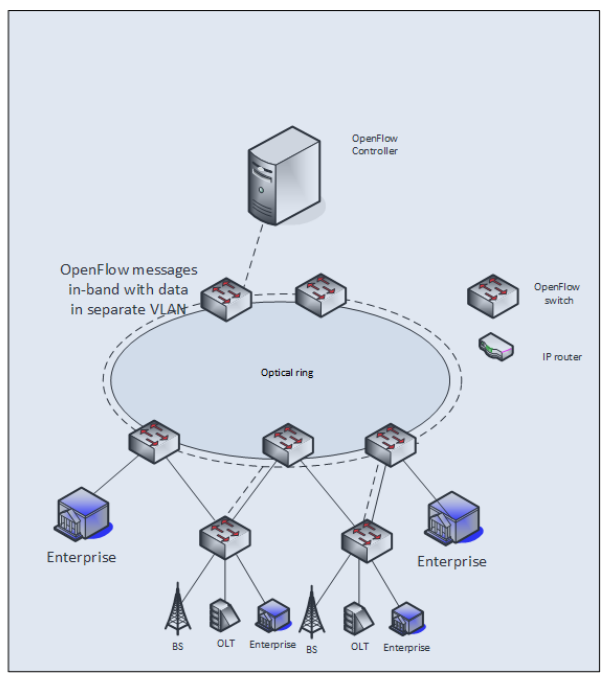

(d)

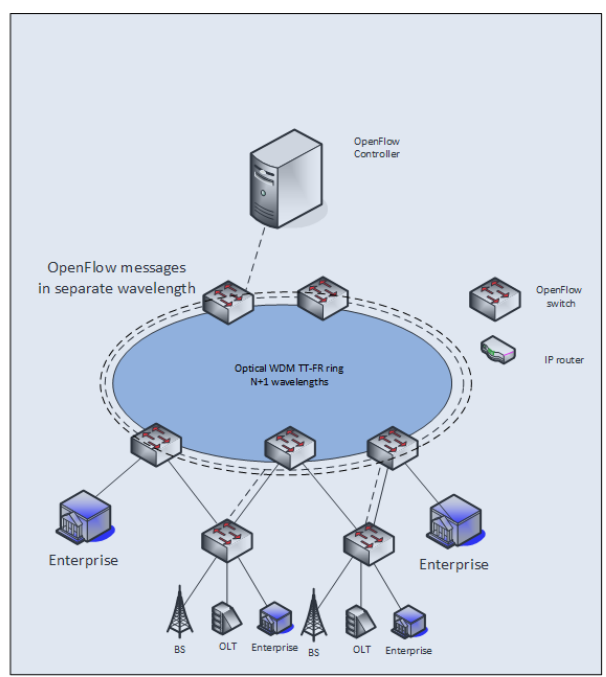

Figure 1. SDN metro architectures with control channel network.

As shown in Fig. 1, there are four metro architectures considered:

- Ring with dedicated out-of-band control infrastructure (Fig. 1-a), requiring dedicated cabling and switches/routers for the control network, which may not be feasible due to extra cost, not only for additional switches and fibers but also for the maintenance of a completely separated network.

- Hop-by-hop ring with in-band control channel and no WDM (Fig. 1-b), which uses in-band connectivity through a non-OpenFlow VLAN (OpenFlow switches can support both non-OpenFlow VLANs and OpenFlow VLANs). In this case, data and control traffic uses the same physical infrastructure (switch port, fiber) but are logically separated with different VLAN IDs. In addition, as an active ring, it requires as many OEO conversions as traversed nodes, more active elements subject to failure and more latency. 
- Optical transparent WDM ring with $\mathbf{N}$ wavelengths (Fig. 1-c): this solution, formerly introduced in [9], proposes the use of WDM TT-FR unidirectional ring to transport layer-2 traffic (Ethernet or OpenFlow), leading to a layer-2 full-mesh topology without OEO conversion. Transparency is achieved because all nodes by-pass all wavelengths except for its dedicated home channel, hence requiring $\mathrm{N}$ wavelengths, one per node. Such a solution uses firstly, an adaptation box to map switch ports to the appropriate wavelengths of a TT-FR WDM ring (for transmission); and secondly, a new adaptation header to differentiate between source nodes (for reception). Finally, it is worth noticing that switches receive both control and data traffic on the same wavelength (in-band), hence there is no physical separation between them. Both data and control coexist on the wavelength, only separated via different VLAN IDs.

- Optical transparent WDM ring with $\mathbf{N}+\mathbf{1}$ wavelengths (Fig. 1-d): this solution is also based on the TTFR WDM ring, using $\mathrm{N}$ wavelengths for data traffic and one additional wavelength to carry control traffic, thus requiring some enhancements to the adaptation box, which are explained in the next section. We call this solution out-of-band since the control traffic is transported over a separated wavelength.

\subsection{Adaptation Box: TT-FR WDM rings with logical full-mesh and $\mathrm{N}+1$ wavelengths}

This approach requires a small enhancement in the adaptation box to deal with the control wavelength, by adding a second fixed receiver. As shown in Fig. 2-a, the switch must allocate the control channel traffic in a separate port in a non-OpenFlow VLAN. This traffic is sent to the adaptation box, which will then be tuned to the specific wavelength dedicated to the control channel (separated from data). On the reception side, two fixed receivers must be used, one for the home channel and another for the control channel. As per Fig. 2-b, in a 5-node ring configuration, six wavelengths will be used, five for the data plane (the figure only shows four) and one for the control plane.

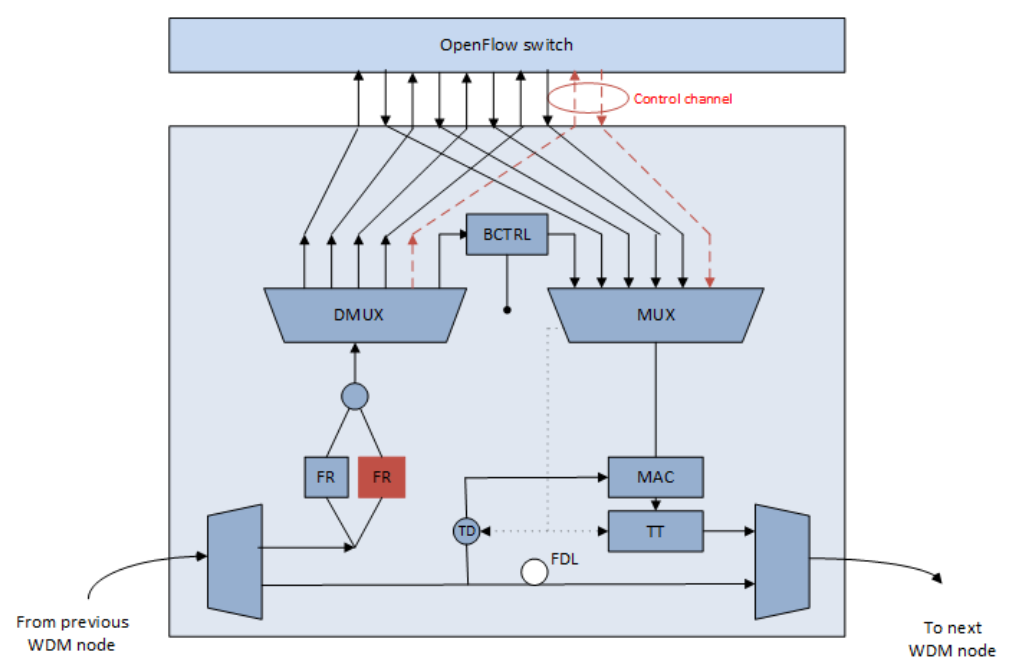

(a)

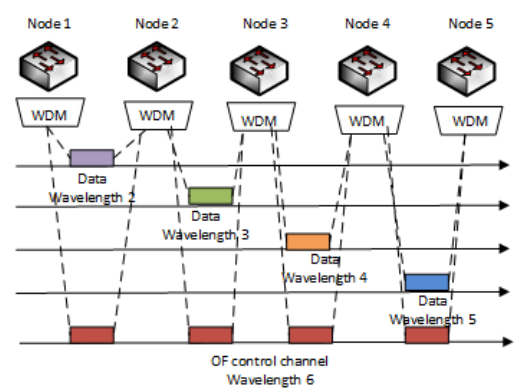

(b)

Figure 2: (a) Architecture of the adaptation box with control channel; b) Control channel and data wavelengths.

\section{COMPARISON}

Table 1 provides a comparison between the four options studied: the first option (Fig. 1-a) has the obvious disadvantage of high cost. Second option (Fig. 1-b) is the cheapest since it does not use either WDM equipment or any adaptation box, however no separation between control and data traffic is provided. Third option (Fig. 1-c) proposes a transparent solution with no OEO conversion, however both data and control traffic coexist on the same logical network. Fourth option (Fig. 1-d) also enables transparency but provides a separate common wavelength for the control traffic. Contention in the shared signalling channel is simply solved by the incoming frame detection before the FDL. 
Table 1. Comparison of the four SDN metro architectures.

\begin{tabular}{|l|c|c|c|c|}
\hline & \multicolumn{1}{c}{$\begin{array}{c}\text { Ring with } \\
\text { dedicated out-of- } \\
\text { band control }\end{array}$} & $\begin{array}{c}\text { Hop-by-hop ring } \\
\text { with in-band } \\
\text { control }\end{array}$ & $\begin{array}{c}\text { Transparent } \\
\text { WDM ring with } \\
\text { N wavelengths }\end{array}$ & $\begin{array}{c}\text { Transparent WDM } \\
\text { ring with } \\
\text { wavelengths }\end{array}$ \\
\hline Cost & $\begin{array}{c}\text { Very high due to } \\
\text { overlay network }\end{array}$ & $\begin{array}{c}\text { Low. Adaptation } \\
\text { box not required }\end{array}$ & $\begin{array}{c}\text { Medium. } \\
\text { Adaptation box }\end{array}$ & $\begin{array}{c}\text { Medium. Enhanced } \\
\text { adaptation box }\end{array}$ \\
\hline WDM required & No & No & Yes: N lambdas & Yes: N+1 lambdas \\
\hline $\begin{array}{l}\text { Extra OEO } \\
\text { conversions }\end{array}$ & 0 & \#hops - 1 & 0 & 0 \\
\hline Data plane & Opaque & Opaque & Transparent & Transparent \\
\hline Control plane & Out-of-band & In-band & In-band & Out-of-band \\
\hline $\begin{array}{l}\text { Reliability of } \\
\text { control plane }\end{array}$ & $\begin{array}{c}\text { Very high if fully } \\
\text { redundant } \\
\text { physical layer }\end{array}$ & $\begin{array}{c}\text { Medium-low. Many } \\
\text { active elements. } \\
\text { Fate sharing with } \\
\text { data plane }\end{array}$ & $\begin{array}{c}\text { Medium-high. Fate } \\
\text { sharing with data } \\
\text { plane }\end{array}$ & $\begin{array}{c}\text { High. Not full fate } \\
\text { sharing with data } \\
\text { plane }\end{array}$ \\
\hline
\end{tabular}

\section{CONCLUSIONS AND FUTURE WORK}

This article has proposed a new SDN-based ring architecture for metro scenarios, where the OpenFlow switches are on top of the TT-FR nodes in the ring. This solution has two main benefits of: (1) transparency in the data plane, i.e. no OEO conversion, and (2) out-of-band control plane in a separate wavelength. The advantage of such an out-of-band solution is that link failures in the data plane may not affect the control network.

For future study, we will consider several aspects to research like the following:

- $\quad 1+1$ protection for the control plane using a second wavelength

- Latency comparison for all four options in a metro environment

\section{ACKNOWLEDGEMENTS}

The authors would like to acknowledge the support of the Spanish projects CRAMnet (grant no. TEC201238362-C03- 01) and FIERRO (grant no. TEC2010-12250-E) to the development of this work.

\section{REFERENCES}

[1] Open Networking Foundation white paper, April 2012: "Software- Defined Networking: The New Norm for Networks", https:/www.opennetworking.org/images/stories/downloads/whitepapers/wp-sdnnewnorm.pdf

[2] OpenFlow Switch Specification, Version 1.3.1, Sept 2012, Open Networking Foundation

[3] M. Casado, T. Koponen, S. Shenker, A. Tootoonchian, "Fabric: A retrospective on evolving SDN", HotSDN 2012, Helsinki, Finland.

[4] N. McKeown, T. Anderson, H. Balakrishnan, G. Parulkar, L. Peterson, J. Rexford, S. Shenker, J. Turner, "OpenFlow: Enabling innovation in campus networks", SIGCOMM CCR, 38(2):69-74, 2008.

[5] D. Staessens, S. Sharma, D. Colle, M. Pickavet, P. Demeester, "Software defined networking: Meeting carrier grade requirements", 2012.

[6] M. Maier, Optical Switching Networks, Cambridge University Press, 2008.

[7] I.M. White et al., "A summary of the HORNET project: A next-generation metropolitan area network", IEEE JSAC, vol. 21, no. 9, Nov. 2003, pp. 1478-94.

[8] N. Bouabdallah, H. Perros, "Cost-effective single-hub WDM ring networks: A proposal and analysis", Comp. Net., vol. 51, no. 13, Sept. 2007, pp. 3878-3901

[9] G. Rodríguez de los Santos et al., "On providing metro Ethernet services over transparent WDM optical rings", IEEE Network, Jan.-Feb. 2011, pp. 14-19. 